Conclusion: Our study demonstrated that RTX is highly effective in children with RD, the majority with SLE, but the safety data obtained indicate the need for careful monitoring of therapy, primarily taking into account the frequency of infections. A decrease in IgG level was observed in a small proportion of pts and did not correlate with the incidence of infections. The frequency of serious infections was low.

Disclosure of Interests: None declared

DOI: 10.1136/annrheumdis-2020-eular.2496

\section{SAT0495 LONG TERM OUTCOME OF JUVENILE IDIOPATHIC ARTHRITIS IN ADULTHOOD; THE MONOCENTRIC EXPERIENCE OF 520 PATIENTS FOLLOWED FOR 20 YEARS IN A TRANSITION TERTIARY CLINIC OF PEDIATRIC RHEUMATOLOGY.}

1. Pontikaki ${ }^{1}$, S. Carbogno ${ }^{2}$, F. Corona ${ }^{2}$, A. Petaccia ${ }^{2}$, R. Cimaz ${ }^{1} .{ }^{1}$ ASST-PINICTO, Chair of Pediatric Rheumatology, University of Milan, MILAN, Italy; ${ }^{2}$ IRCCS Cà GRANDA Ospedale Maggiore Policlinico, MILAN, Italy

Background: Juvenile Idiopathic Arthritis (JIA) is a chronic pediatric inflammatory disease that shows many differences compared to adult-onset arthritis. The different clinical manifestations, the assessment and the management of JIA is the reason that the transition from childhood to adulthood is an important multidimensional process that emphasizes a lot of aspects.

Objectives: To describe the long-term outcome of JIA

Methods: Five-hundred and twenty patients affected by JIA and referred to a transition care rheumatology tertiary centre were considered between 1999 and 2019. The outcome assessment included remission, disease duration, medications, number of prosthesis implantation, pregnancies, mortality and social integration (employment status and educational level).

Results: A hundred and thirty-eight (26\%) males and $382(73 \%)$ females were included; $157(30 \%)$ patients were lost to follow up. The mean age of the patients was $27(18-57)$ years, with a mean age at onset of 8 years and an average disease duration of 19 years. Subtypes of JIA at disease onset included 252 (48\%) oligoarthritis, $134(26 \%)$ polyarthritis, $64(12 \%)$ systemic arthritis, $22(4 \%)$ psoriatic arthritis, $43(8 \%)$ enthesitis related arthritis and 1 $(0.1 \%)$ undifferentiated arthritis. Ninty-three $(18 \%)$ patients suffered of uveitis. Ninty-five implant prosthesis and 16 arthrodesis were recorded. At follow up $198(38 \%)$ patients were on remission of which $107(20 \%)$ off medication. Among the 322 patients still on medication, 84 (16\%) were under treatment with oral steroids, 226 (43\%) with sDMARDs and 249 (40\%) with bDMARDs. Five deaths (1\%) occurred in this cohort. Two hundred and thirty-five subjects had a higher educational level, 327 had an employment. We have data of twenty-nine pregnancies. The transition age was considered after the age of 16 years old. The key word for the management of this cohort was the multidisciplinary approach towards each patient, with the collaboration of other specialists (ophthalmologist, orthopedic, dermatologist, gastroenterologist, obstetric and psychologist).

Conclusion: In the era of biologic therapy the long-term outcome of JIA underwent an outstanding improvement regarding a lot of variables. Two hundred and thirty-two patients were still followed, not only because of the continuation of the biological therapy, but also for a multidisciplinary care even during remission. JIA often persists over the adulthood, therefore the long term follow-up and care of these patients needs to be conducted by a rheumatologist expertized in JIA in collaboration with other specialists.

Disclosure of Interests: None declared

DOI: 10.1136/annrheumdis-2020-eular.5540

\section{SATURDAY, 06 JUNE 2020}

\section{Basic and translational science in paediatric rheumatology}

\section{SAT0496 \\ INTERROGATING THE CIRCULATORY IMMUNE ARCHITECTURE OF ENTHESITIS-RELATED ARTHRITIS}

S. H. Tay ${ }^{1,2}$, J. Y. Leong' ${ }^{2}$, M. Wasser ${ }^{2}$, A. J. M. Lim², P. Chen², J. G. Yeo ${ }^{1,2,3}$, T. Arkachaisri ${ }^{1,4}$, S. Albani ${ }^{1,2,3}{ }^{1}$ Duke-NUS Medical School, Singapore, Singapore; ${ }^{2}$ Translational Immunology Institute, SingHealth/Duke-NUS Academic Medical Centre, Singapore, Singapore; ${ }^{3} K K$ Women's and Children's Hospital, Singapore, Singapore; ${ }^{4} K K$ Women's and Children's Hospital, Rheumatology \& Immunology Service, Singapore, Singapore
Background: Enthesitis-related arthritis (ERA) is one of the most common subtype of juvenile idiopathic arthritis (JIA) in Asia ${ }^{1}$. It carries a poor prognosis, but limited knowledge of its pathogenesis hampers clinical diagnosis and treatment. Objectives: We hypothesise multiple aberrations from the healthy immunome culminating in an imbalance between the immune effector and regulatory cel subsets as key for driving ERA pathogenesis. Thus, we employed a comprehensive high-dimensional interrogative strategy using mass cytometry to assess the ERA immune architecture ${ }^{2}$

Methods: We examined peripheral blood mononuclear cells from 30 ERA patients (15 with active sacroilitis, 15 without active sacroilitis) within the first two years of disease and 30 healthy paediatric controls with mass cytometry, using two extensive antibody panels encompassing key lineage and functiona markers. Dimensional reduction and unsupervised clustering were performed to identify immune cell subsets differentially present in ERA patients. Manual gating was performed to further describe observed differences in subset frequencies. These subsets were statistically evaluated with reference to the healthy cohort and their association with disease activity determined.

Results: We identified broad differences in the ERA circulatory immune architecture that involved both innate and adaptive immune cell populations, notably with the enrichment of naive CD4+ T cells as well as depletion of cytolytic NK cells (CD56 ${ }^{\text {dim }}$ CD16+). The chemotactic profiles of their subsets also differed in ERA patients, which underscores their migratory capacity and hence potential effector role in the ERA arthritic microenvironment. In addition, there were some dissimilarities in the circulatory immunome of ERA patients with active sacroiliitis as compared to those without, which alludes to a possible mechanistic basis behind the disease complication.

Conclusion: This is the first study, via deep parameterisation afforded by mass cytometry, to demonstrate a concomitant dysregulation of both innate and adaptive immune cell subsets in ERA patients. Further mechanistic studies of these immune cell subsets and their functional networks will inform the development of diagnostic and prognostic markers that can reliably predict clinical fate in ERA, thereby complementing clinical assessment in the care of ERA patients.

References:

[1] Arkachaisri et al. Paediatric rheumatology clinic in Southeast Asia: are we different? Rheumatology (Oxford). 2017.

[2] Tay et al. Immunomics in pediatric rheumatic diseases. Front Med (Laus anne). 2019.

Disclosure of Interests: None declared

DOI: 10.1136/annrheumdis-2020-eular.5125

\section{SAT0497 \\ A PILOT PROTEOMIC ANALYSIS OF PLASMA BIOMARKERS IN IGA VASCULITIS}

S. Demir ${ }^{1}$, M. Sardan ${ }^{2}$, I. Yet ${ }^{3}$, E. Sag ${ }^{1}$, Y. Bilginer ${ }^{1}$, Ö. Celikbıcak ${ }^{4}$, S. Özen ${ }^{1}$. ${ }^{1}$ Hacettepe University Faculty of Medicine, Department of Pediatric Rheumatology, Ankara, Turkey; ${ }^{2}$ Hacettepe University Faculty of Medicine, Hunitek Research Center, Ankara, Turkey; ${ }^{3}$ Hacettepe University Faculty of Medicine, Department of Bioinformatics, Ankara, Turkey; ${ }^{4}$ Hacettepe University Faculty of Medicine, Department of Chemistry, Ankara, Turkey

Background: IgA vasculitis/ Henoch Schönlein Purpura (IgAV/HSP) is the most common vasculitis of childhood, characterized by the $\lg \mathrm{A} 1$ immune deposits in the small vessels. Although it is very common, the understanding of its molecular pathogenesis is still very limited.

Objectives: We aimed to analyse plasma proteomes of IgAV/HSP patients using nano liquid chromatography - tandem mass spectrometry (nLC-MS/MS) to investigate the disease pathogenesis.

Methods: IgAV/HSP was diagnosed according to the Ankara criteria in 2008 (1). Five active IgAV/HSP patients and two age and gender-matched health controls were enrolled in this pilot study. Serum samples from subjects were collected on the same day of IgAV/HSP diagnosis and before steroid or other immunosuppressive treatment initiated. Sample preparation was carried ou using PreOmics iST Kit. We investigated the alteration of serum proteome using the nano LC-MS/MS approach. Bruker raw files were analyzed using the proteomics software Max Quant (1.6.7.0). The human reference proteome set from UniProt was used to identify proteins. Proteomic data were analyzed with Perseus 1.6.7.0.

Results: The data file includes peptide and protein identification, accession numbers, protein and gene names, sequence coverage and label free quantification (LFQ) values of each sample. 345 proteins were reported per sample. Identifications from the reverse decoy database, identified by site only and known contaminants were excluded. Data were log transformed. Two sample T-test was performed between groups. We identified 23 significantly different expressed proteins (Table 1). Mainly the differentially expressed proteins were in the $\mathrm{Ig}$ and complement pathway, innate immune inflammatory, 